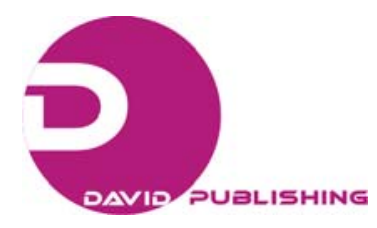

\title{
Risk Management: A New Project Management Perspective
}

\author{
Pasis Mselle $^{1}$, Jacob Kashiwagi ${ }^{2}$, Dean Kashiwagi ${ }^{2}$ and Aderemi Adeyemi ${ }^{1}$ \\ 1. Project Management Section, Civil Engineering Department, University of Botswana, Botswana \\ 2. Performance Based Studies Research Group (PBSRG), Arizona State University, USA
}

\begin{abstract}
Risk management in Botswana has been very ineffective at assisting projects to be on time, on budget, and meeting client's/buyer's expectations. Traditional risk management attempts to manage, control, and direct a project through various phases, from planning and design, through procurement, and to construction. However, the risk management in Botswana seems to be either not implemented or not successful. The project performance in Botswana has a poor performance record. The researchers are attempting to identify why the traditional project/risk management is not working. In analyzing the problem in Botswana, the researchers discovered that the traditional risk management model was theoretically unsound, and designed a new project and risk model, with an entirely different approach to risk. The new model is an outgrowth of the highly successful Performance Information Procurement System (PIPS) and the Information Measurement Theory (IMT)/Kashiwagi Solution Model (KSM) concepts. The approach has been presented to some Botswana clients and academics and has received a favorable response. The development of the new risk model will lead to a huge change in the paradigm for delivering projects in Botswana. The theoretical development of the new risk model is ongoing at the University of Botswana as a part of a doctoral dissertation.
\end{abstract}

Key words: Risk management, Botswana projects, new leadership based PM model.

\section{Introduction}

Botswana is a developing country that depends on project management expertise and traditional models from the UK, Europe, and the United States. Due to the lack of experience and expertise, practitioners in Botswana are looking for simple models that can result in successful project delivery. An effort is underway at the University of Botswana (UB) Project Management section to define risk, project management, and project delivery in simplistic terms. They have been assisted by a Fulbright Scholar and research professor from the Performance Based Studies Research Group (PBSRG) at Arizona State University, the home location of the PMTomorrow and CIB W117 journal that looks at futuristic systems that are more simplistic and successful.

Corresponding author: Dean Kashiwagi, $\mathrm{PhD}$, professor, research fields: procurement, risk management, project management, and supply chain management. E-mail: Dean.Kashiwagi@asu.edu.
Risk is defined in project management as something that is unexpected or not predicted that is a negative deviation from buyer/user expectation [1]. Risk can be measured in terms of cost, time, and customer dissatisfaction. It is commonly perceived as being created by nonperforming vendors who do not deliver what is expected. Risk management (RM) or risk minimization is normally the responsibility of project managers (PM) representing the buyer. Risk is a negative term that speaks poorly of a project manager's capability to manage a project [2-3].

The traditional method of risk management (RM) by the client's project manager (PM) in Botswana is to [4-6]:

(1) Ensure that the buyer knows exactly "what" they want, ensure that the buyer has the technical expertise (or hires a professional) to create the directions, and to manage, direct, and control a vendor to get the expected outcome. 
(2) Use a well written technical specification/contract to ensure that the vendor meets the expectations of the buyer for the budgeted amount in the time required.

(3) Maximize the flow of information and interface between the project manager, users and the vendors. This includes ensuring that the owner verifies the costs in detail of the competing vendors.

The buyer's PM is required to [4-7]:

(1) Be able to appraise the impact of the service in terms of expectation, monitor the service, and evaluate the performance and value of the service.

(2) Know more than the vendor in terms of technical requirements, quality, details, delivery, and cost.

(3) Write a specification/contract that identifies the buyer's expectations in terms of technical requirements.

(4) Be able to inspect/regulate the vendor to meet contract requirements.

(5) Ensure they have a complete cost breakdown of the vendor.

(6) Ensure that they know what is happening at all times through constant measurement and information.

(7) Manage and minimize the risk of change orders.

(8) Ensure that the buyer gets the services it needs for the best price.

The client's representatives (project manager, quantity surveyor, and procurement agent) are the risk managers of the project. They identify the requirement and manage, control and direct the contractor. They do not do quality control, because quality control is defined as being performed by the vendor's personnel doing the project [8].

\section{Existing Risk Management Model}

The existing risk management model is shown in Fig. 1. The buyer's PM identifies a budget, an expected service, and a time period of delivery. They do not know enough to know if their budget and time to deliver is accurate unless they are actually doing the work and have technical expertise that is current, have dominant measurements of past similar projects, and a knowledge of the current industry supplier costs and capability. Rarely does the buyer's PM have the accurate information of the initial conditions at the beginning of the event.

Instead, they make decisions based on best guess due to incomplete information. They then hire a technical expert professional who makes more decisions on how the expectation can be met by a vendor, by writing a specification. A procurement agent then competes the vendors based on price, assuming that all the vendors and personnel are the same, and usually select the lowest priced vendor. The PM then attempts to manage and control (M\&C) the risk by controlling, directing, and inspecting the vendor's work. They often seek the assistance of a quantity surveyor or engineer, who controls the contractor's amounts and prices. The problem with this risk management model is that the client, client's PM, and procurement agent are making decisions to determine the requirements and expected vendor service. It may not be complete or an accurate

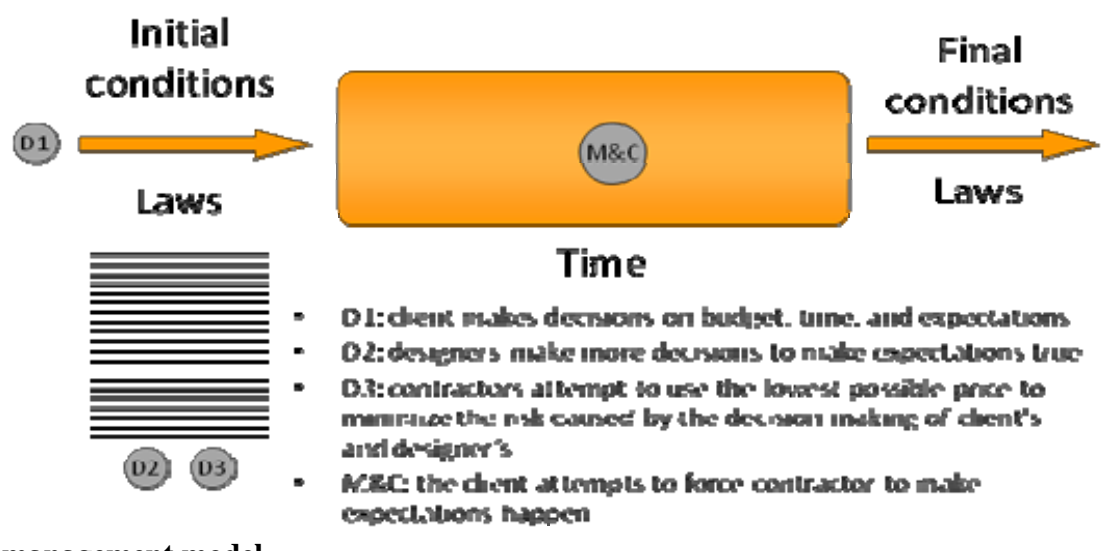

Fig. 1 Existing risk management model. 
perception of the initial conditions of the project or event (accurate and complete view of reality.) The client's team then attempts to control the contractor to achieve the expected outcome.

Through deductive analysis, this method of project delivery and risk management has the following potential weaknesses [9-12]:

(1) Expert hires the non-expert to do work. It proposes that a PM or consultant can know more about delivering a service than the expert vendors who deliver the service. Or it is assuming that it is logical for someone who is an expert but who lacks sufficient information to hire a vendor who knows less than them.

(2) Control. A buyer can use a contract to force/control expected performance from the lowest bidding vendor. The concept of control has never proven successful.

(3) Non-expert can understand everything. A PM representing the client can understand all the information that the expert vendor is using and can control and direct the vendor. Unless the PM is the expert who can do the work, in which case the question should be asked why they aren't doing the work, how can they be more expert than the vendor who does the work for their core expertise?

(4) Vendors are the same. Multiple individuals can read a complex, detailed, technically oriented document that is rarely perfect and flawless, and perfectly understand what the writer of the document is expecting, and deliver it in the exact manner, time and cost that the writer envisioned.

(5) Win-lose can work. The buyer can take the low priced alternative and then influence or control the vendor, who perfectly understood what everyone else did, to meet the expectations of the buyer.

(6) Management is an effective and efficient practice. Management, direction, and control is an efficient and effective method of delivery of a technically based expert service.

This is a high risk, risk management model that goes against all the deductive logic of Information
Measurement Theory (the measurement of information to predict the future outcome) and common sense [11, 13]:

(1) Decision making is the source of risk. Buyer's PM makes decisions on what can be provided without knowing if it is possible or being able to provide the services themselves.

(2) The need for management, direction, and control shows inefficiency and poor performing personnel. It increases management activity (direction, control, and inspection) and expects higher value.

(3) Influence. Assume it is possible for one party to control or influence another party.

(4) Hire non-performing vendors to do work. Assume that a buyer's project manger is the expert and just needs manpower.

(5) Assume that risk is being caused by the expert vendor. Or else why would someone hire an inexperienced vendor who must be managed.

Whatever combination of factors, this risk model does not meet the test of logic and common sense and goes against dominant facts of reality. What is the source of risk and how should it be minimized?

\section{The Event Model}

The event model shown in Fig. 2, has the following assumptions [14]:

(1) Initial conditions, the event period, and final conditions.

(2) Laws of physics, nature, logic and reality are able to predict how conditions will change over time.

Once the initial conditions are fixed (physical environment, people, and laws), someone with all information can predict the future outcome. The more accurate the perception of the initial conditions, the more accurate the prediction will be of the final outcome. If the laws of logic and laws apply, someone should be able to explain why the event outcome occurred once given the information on the outcome and the initial conditions (called hindsight). 
Initial

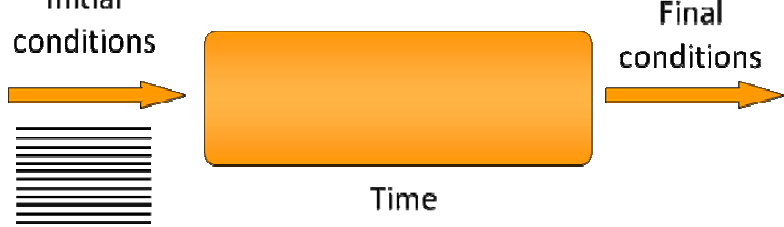

Fig. 2 The event model.

Kashiwagi [15] proposes that events happen only one way. Once the initial conditions are fixed, the event cannot be controlled to change the final outcome to something which is not related to the initial conditions. A change in the initial conditions will result in a change of the final conditions. The initial conditions and final conditions are different only by the operation of laws which is predictable given all information. The lack of information does not change the final outcome. Kashiwagi also proposes the following:

(1) There is no such thing as randomness and chance. This only is perceived as true when information is lacking, or the people cannot perceive the information.

(2) Randomness is an assumption used by people who lack information to determine a good guess of what is existing.

Kashiwagi proposes that Einstein's perception of reality was more accurate than Heisenberg's [16]. Kashiwagi [11] acknowledges that Heisenberg's concepts assist in identifying what is going on when there is a lack of information. Therefore, Kashiwagi [11] proposes that Hawking's approach of identifying where randomness and deterministic behavior apply is one being too focused on what he doesn't know, and not on logic and what he does know.

The event model identifies that risk occurs because people do not perceive all the information on the initial conditions. Human nature has led people to then make decisions, have expectations, and attempt to control the event. In all cases where people have dominant information, it has been identified that people cannot be controlled by other people. Dominant examples of this include:

(1) Prohibition.
(2) Drug war.

(3) Rehabilitating hardened criminals in prison.

(4) Foreign policy in Vietnam, Korea, Iraq, Afghanistan, Somalia, Haiti, Soviet Union, China, and North Korea.

(5) Welfare program.

(6) Minority contracting, small business, and federal government 8A programs.

(7) Poor delivery of construction and IT services.

\section{Source of Risk}

Using the event model, risk is defined by the authors as when someone inaccurately perceives the initial conditions. Decisions are then made to compensate for the lack of information. Expectations of the final outcome are then created from the inaccurate perception of the initial conditions. When the event is happening, the conditions constantly change based on the previous instant (concept of differentials, rate of change, or finite method analysis.) When the reality of the event conditions at a specific time differs from the expectation of someone who did not perceive the accurate conditions, the differential is identified as risk, and someone is hired to manage and control the risk.

Simply put, risk is caused by decision making in the initial conditions that results in inaccurate expectations. Unless the client's PM can accurately identify the initial conditions (vendor's capability to deliver an expected service), and therefore predict the future outcome, the PM's decision making is creating risk.

Risk is minimized by the following activities:

(1) Finding the individuals who can most accurately identify the initial conditions (expert).

(2) Allowing the people who can identify the initial conditions and can identify if an individual's expectations can be done (expert).

(3) Minimize the attempt to manage, direct, and control the event by preplanning and using experts.

(4) Allowing the expert to control their own event, because they are the expert who knows what is there, and what to do, and how to get to a realistic outcome. 


\section{Decision Making}

People make decisions when they have insufficient information and don't know what is going to happen. If they understood enough dominant information of the initial conditions, which is simple, understood by all, and will predict the outcome without decision making, risk is minimized. Decisions are made when we do not know what will happen. Decisions are made in the following environments:

(1) The situation seems complex.

(2) "Experts" come in to make decisions. This is a paradox, because experts minimize decision making.

(3) People disagree on the perception of the initial conditions.

(4) The decision makers lack critical information.

(5) The decision makers do not have liability and accountability for what happens.

(6) Decision makers are usually management personnel, and not the people who will do the service.

When decision making goes up, risk goes up. Decision making is done by people who lack information. Decision making is the source of risk.

Instead of being an efficient and effective risk management model, this is a risk making model. The authors propose it is this risk management model of making decisions, managing, directing, and controlling which causes risk and the poor performance of the delivery of services in Botswana. The authors also propose that this risk management model also is being ineffectively and inefficiently used in delivering construction and IT services in the United States and other developed countries. A new risk management model is required.

\section{New Risk Management Model}

The new risk management model will have the following characteristics:

(1) Decision making should be minimized.

(2) If expectations are caused by decision making due to the lack of information, the expectations must be corrected by experts who provide the service, who can define the difference between the expectation and reality, and who can take accountability to deliver the reality based outcome.

(3) Experts should not make decisions. Experts should be able to predict an event from beginning to end. Experts would do their most critical work before the event.

(4) The expert must be given control and responsibility to manage and control the risk that they do not control. If any other participant tries to change the initial conditions requirement or preplan to deliver the service, the vendor must have control to identify the risk, document the risk, and to minimize the impact of the risk.

(5) The risk management model must motivate all participants to understand the initial conditions, and be accountable to do what is agreed upon in the initial conditions.

(6) No managers should direct the experts.

(7) The experts should identify what the final conditions should be.

Transition from the traditional risk management model to the new model should include the following changes:

(1) More time should be spent before the event than during the event.

(2) The identification that it is the client and their representatives and not the vendors who are causing risk.

(3) A methodology to find experts without being experts is required.

(4) Control of the project should be transferred to the expert.

(5) What a buyer/client expects should be replaced by what an expert can actually deliver.

Therefore, the new risk management model is where the expert vendor minimizes the risk with their expertise using a quality control/risk management model, and the client's PM minimizes risk by:

(1) Assuming that the client's perception on the requirement and constraints is not totally accurate. 
(2) Assuming that there are expert vendors who because of their expertise have a more accurate perception of the requirement and constraints.

(3) Use a delivery structure that assume all vendors to be experts and require them to act like experts. They should easily justify their expertise through dominant measurements, identify the risk that other competitors have because they are not experts and explain why they do not have the risk, identify risk that they do not control which includes client's false expectations, constraints of existing conditions, and risks caused by people who they do not control, and how they will manage and minimize those risks, and to predict the future with a baseline plan that they can be accountable to.

(4) Select the best value that minimizes the risk by identifying the most accurate assessment of initial conditions and the future outcome.

(5) Allow the expert vendor to preplan, identify the risks, and manage and minimize the risk that they do not control before they start.

(6) Force the expert to document the deviations from their baseline plan, identifying and justifying the deviations.

(7) The best value vendor's more accurate perception of the project, replaces the client's best guess requirement.

This new model will allow the following issues to be resolved:

(1) Client PMs do not have to be technical experts on what they are delivering.

(2) Budget and scope requirements do not have to be perfect.

(3) Decisions and impact of decisions are minimized.

(4) If decisions are made, they are made by the experts, minimizing risk.

(5) Control and risk can be transferred to the best value vendor.

(6) Quality control can be performed by the vendor who is documenting, measuring, and minimizing deviation instead of “someone’s perception”.

(7) Vendors are accountable because the proposal is their vision.

(8) PMs can do quality assurance (ensuring that best value selection is done, ensuring that vendor has system to manage and minimize risk).

(9) Forces vendors to have technical expertise, use preplanning to minimize risk, and manage their own projects.

\section{Paradigm Shift}

More than a legal, procurement or project management model, the new risk management model is a change of paradigm. It is a movement by the PM from being the expert, to identifying who the expert is, and forcing the expert to act like the expert. It is moving from a technical approach by the PM, to a logic, common sense, holistic approach. It is moving from trusting the decision making of a PM to a structure that forces the vendors to look ahead, to measure their performance, to set a baseline project scope and schedule, and measure deviation from the baseline plan. It is a movement from management, direction, and control to forcing the contractor to do risk management and quality control, and where the PM does quality assurance. It is movement from management and control to enabling, best value practices, and leadership.

It is a movement from being the center of the universe, the ultimate knowledge center, to a position of knowing "that we don't know". It is seeking experts, instead of using the PM's expertise.

The new PM responsibilities will be:

(1) Identifying the client/buyer's expectation.

(2) Identifying the best value vendor by assuming that they are all experts.

(3) Assisting the expert vendor to prepare the final contract documents.

(4) Doing quality assurance (ensure that the expert vendor is doing quality control and risk management and managing by the minimization of time, cost, and 
customer satisfaction/expectation deviation).

\section{Conclusion}

This research project which uses deductive analysis identifies the buyer and the buyer's PM as the main source of risk to any project or delivery of services. It identifies decision making due to the lack of information. that creates a false expectation which results in risk. It proposes that the difference between reality and false expectations is risk. The risk is therefore generated by the client/buyer, and not the vendors. Hiring a vendor who is technically not qualified maximizes the problem. Further risk is generated by the client's PM attempting to manage and control the vendor, increasing the flow of information and decision making, and becoming more and more important as the confusion grows.

The new risk management model states that the following must be done to minimize risk:

(1) Assume that the client's perception on the requirement and constraints is not totally accurate.

(2) Assume that there are expert vendors who because of their expertise have a more accurate perception of the requirement and constraints.

(3) Use a delivery structure that assumes all vendors to be experts and require them to act like experts. They should easily justify their expertise through dominant measurements, identify the risk that other competitors have because they are not experts and explain why they do not have the risk, identify risk that they do not control which includes client's false expectations, constraints of existing conditions, and risks caused by people who they do not control, and how they will manage and minimize those risks, and to predict the future with a baseline plan that they can be accountable to.

(4) Select the best value that minimizes the risk by identifying the most accurate assessment of initial conditions and the future outcome.

(5) Allow the expert vendor to preplan, identify the risks, and manage and minimize the risk that they do not control before they start.

(6) Force the expert to document the deviations from their baseline plan, identifying and justifying the deviations.

(7) The best value vendor's more accurate perception of the project, replaces the client's best guess requirement.

The new risk management model can also be defined by the following terms:

(1) Outsourcing: using the expertise of the vendor.

(2) Empowering.

(3) Best value.

(4) Allowing experts to minimize risk through use of their expertise.

(5) Preplanning.

(6) Alignment of resources.

(7) Minimization of management, control, direction, decision making, and risk.

(8) Documentation of risk and risk mitigation (risk management plan and weekly risk reports).

(9) Quality control (by contractor) and quality assurance (by vendor).

(10) Measured environment.

The new risk management model transfers the minimization of risk to a structure called the Performance Information Procurement System/ Performance Information Risk Management System (PIPS/PIRMS) and technically expert vendors. The new RM model uses logic, simplicity, and finds experts who know why they are experts, and who can predict the future outcome and use dominant performance measurements.

It minimizes the activity of PMs, and allows them to be successful, visionary and proactive instead of being reactive and continually putting out fires.

The new risk management model has been developed in parallel with the testing of the concepts in the PIPS/PIRMS systems tests. It has been implemented due to the dominant logic and deductive nature of the principles in the United States, Netherlands, and has been used in some tests in 
Botswana. However, the new risk model must be completely documented and defined before the traditional PM practitioners can use the concept. This task is being accomplished by a $\mathrm{PhD}$ dissertation by Pasis Mselle, one of the authors at the University of Botswana Project Management Section.

\section{References}

[1] S. Ward and C. Chapman, Transforming project risk management into project uncertainty management, International Journal of Project Management 21 (2) (2003) 97-105.

[2] G. Angel, PMP Certification: A Beginner's Guide, McGraw-Hill Companies, 2010.

[3] C. Chapman and S. Ward, Project Risk Management: Processes, Techniques and Insights, John Wiley \& Sons Ltd., 1997.

[4] D. K. Chimwaso, An evaluation of cost performance of public projects: A case of Botswana, in: Proceedings of 2nd International Conference of CIB TG 29 on Construction in Developing Countries, Gaborone, Botswana, 15-17 Nov., 2000.

[5] J. K. Ssegawa and A. B. Ngowi, Causes of Poor Performance of Public Construction Projects in Botswana, Department of Civil Engineering, University of Botswana, Garborone, 2007.

[6] J. K. Ssegawa, Prevailing financial management practices by small and medium scale firms in Botswana, in: Proceedings of 2nd International Conference of CIB TG
29 on Construction in Developing Countries, Gaborone, Botswana, 15-17 Nov., 2000.

[7] E. Sabaa, The skills and career path of an effective project manager, International Journal of Project Management 19 (1) (2001) 1-7.

[8] E. W. Deming, Out of the Crisis, Cambridge, Massachusetts: Massachusetts Institute of Technology, 1985.

[9] G. Bradshaw, Establishing a first class project controls organization for managing large complex projects, TC11-20, 2008.

[10] M. Casinelli, Guidelines to mitigate schedule delay-from the owner's viewpoint, Cost Engineering 47 (2) (2005) 21-27.

[11] D. T. Kashiwagi, A Revolutionary Approach to Project Management and Risk Management. Performance Based Studies Research Group, Tempe, AZ, 2010.

[12] B. B. Lepatner, Broken Buildings, Busted Budgets, The University of Chicago Press, Chicago, United States of America, 2007.

[13] J. Kashiwagi, Leadership is alignment not influence, Master Thesis, Arizona State University, Tempe, AZ: United States of America, 2007.

[14] D. T. Kashiwagi, Best Value Procurement (IMT, KSM\& PIPS) (3rd ed.), 2008.

[15] A. Adeyemi, D. Kashiwagi and K. Sullivan, Acceptance of IMT/PIPS philosophy in Botswana, in: RICS COBRA Research Conference, University of Cape Town, 10-11th Sep., 2009, pp. 509-521.

[16] W. Heisenberg, Physics and Philosophy: The Revolution in Modern Science, HarperCollins Publishers, 2007. 